\title{
ABM Clinical Protocol \#7: Model Breastfeeding Policy (Revision 2010)
}

\author{
The Academy of Breastfeeding Medicine Protocol Committee
}

A central goal of The Academy of Breastfeeding Medicine is the development of clinical protocols for managing common medical problems that may impact breastfeeding success. These protocols serve only as guidelines for the care of breastfeeding mothers and infants and do not delineate an exclusive course of treatment or serve as standards of medical care. Variations in treatment may be appropriate according to the needs of an individual patient.

\section{Purpose}

$\mathbf{T}$ HE PURPOSE OF THIS PROTOCOL is to promote a philosophy and practice of maternal-infant care that advocates breastfeeding. Care should support the normal physiologic functions involved in the establishment of this maternalinfant process and assist families choosing to breastfeed with initiating and developing a successful and satisfying experience.

This policy is based on recommendations from the most recent breastfeeding policy statements published by the Office on Women's Health of the U.S. Department of Health and Human Services, ${ }^{1}$ the American Academy of Pediatrics, ${ }^{2}$ the American College of Obstetricians and Gynecologists, ${ }^{3}$ the American Academy of Family Physicians, ${ }^{4}$ the World Health Organization, ${ }^{5}$ the Academy of Breastfeeding Medicine, ${ }^{6}$ and the UNICEF/World Health Organization evidence-based Ten Steps to Successful Breastfeeding. ${ }^{5,7-10}$

In addition to evidence supporting each of the Ten Steps improving breastfeeding exclusivity or duration, there is also documentation of a dose-responsive effect: Women at hospitals implementing six of seven studied steps in one report were six times more likely to meet their exclusive breastfeeding goals than those from hospitals implementing no or only one of the steps. ${ }^{11}$ The degree of compliance is also important: Breastfeeding duration is longer when hospitals' selfreported compliance with the steps is better. ${ }^{12}$

\section{Policy Statements}

1. The "name of institution" staff will actively support breastfeeding as the preferred method of providing nutrition to infants. A multidisciplinary, culturally appropriate team comprising hospital administrators, physician and nursing staff, lactation consultants and specialists, nutrition staff, other appropriate staff, and parents shall be established and maintained to identify and eliminate institutional barriers to breastfeeding. On a yearly basis, this group will compile and evaluate data relevant to breastfeeding support services and formulate a plan of action to implement needed changes.

2. A written breastfeeding policy will be developed and communicated to all healthcare staff. The "name of institution" breastfeeding policy will be reviewed and updated biannually using current research as an evidence-based guide.

3. All pregnant women and their support people as appropriate will be provided with information on breastfeeding and counseled on the benefits of breastfeeding, contraindications to breastfeeding, and risk of formula feeding. ${ }^{13}$

4. The woman's desire to breastfeed will be documented in her medical record.

5. Mothers will be encouraged to exclusively breastfeed unless medically contraindicated. The method of feeding will be documented in the medical record of every infant. (Exclusive breastfeeding is defined as providing breastmilk as the sole source of nutrition. Exclusively breastfed babies receive no other liquids or solids with the exception of oral medications prescribed by a medical care provider for the infant.)

6. At birth or soon thereafter all newborns, if baby and mother are stable, will be placed skin-to-skin with the mother. Skin-to-skin contact involves placing the naked baby prone on the mother's bare chest. The infant and mother can then be dried and remain together in this position with warm blankets covering them as appropriate. Mother-infant couples will be given the opportunity to initiate breastfeeding within 1 hour of birth. Post-cesarean-birth babies will be encouraged to breastfeed as soon as possible, potentially in the operating room or recovery area (Table 1). The administration of vitamin $\mathrm{K}$ and prophylactic antibiotics to prevent ophthalmia neonatorum should be delayed for the first hour after birth to allow uninterrupted motherinfant contact and breastfeeding. ${ }^{14-16}$

7. Breastfeeding mother-infant couples will be encouraged to remain together throughout their hospital stay, 
Table 1. Best Practices for Breastfeeding Support Following Cesarean Delivery

Early mother-infant contact. Avoidance of separation unless dictated by medical indications

Early breastfeeding $<1$ hour after delivery. Can occur in delivery suite or recovery room

Regional anesthesia for cesarean delivery

Infant positioning to minimize incision discomfort. Use of side-lying, football breastfeeding position. Use of pillow to protect incision site

Use of regional medication after cord clamping to decrease the need for postoperative narcotics

Preferential use of narcotics with less adverse effects on neonatal behavior

Frequent breastfeeding and rooming-in such as would be routine for vaginal delivery

Protocols for early breast pumping and expression if infant separation is dictated because of medical indication such as

prematurity. Should be initiated day of delivery

Easy availability of lactation expert for further support and assistance if needed

Monitoring for delayed onset of lactation in mother and excessive weight loss in the newborn

Education and encouragement of family members in methods of supporting breastfeeding in the new family

including at night (rooming-in). Skin-to-skin contact will be encouraged as much as possible.

8. Breastfeeding assessment, teaching, and documentation will be done on each shift and whenever possible with each staff contact with the mother. Each feeding will be documented, including latch, position, and any problems encountered in the infant's medical record. For feedings not directly observed, maternal report may be used. Every shift, a direct observation of the baby's position and latch-on during feeding will be performed and documented.

9. Mothers will be encouraged to utilize available breastfeeding resources, including classes, written materials, and video presentations, as appropriate. If clinically indicated, the healthcare professional or nurse will make a referral to a lactation consultant or specialist for additional education and assistance.

10. Breastfeeding mothers will be instructed about:

a. Proper positioning and latch-on

b. Nutritive suckling and swallowing

c. Milk production and release

d. Frequency of feeding/feeding cues

e. Hand expression of breastmilk and use of a pump if indicated

f. How to assess if infant is adequately nourished and

g. Reasons for contacting the healthcare professional

These skills will be taught to primiparous and multiparous women, provided in written form, ${ }^{17}$ and reviewed before the mother goes home.

11. Parents will be taught that breastfeeding infants, including cesarean-birth babies, should be put to breast a minimum of eight to 12 times each 24 hours, with some infants needing to be fed more frequently. Infant feeding cues (e.g., increased alertness or activity, mouthing, or rooting) will be used as indicators of the baby's readiness for feeding. Breastfeeding babies will be breastfed at night.

12. Time limits for breastfeeding on each side will be avoided. Infants can be offered both breasts at each feeding but may be interested in feeding only on one side at a feeding during the early days.

13. No supplemental water, glucose water, or formula will be given unless specifically ordered by a healthcare professional (e.g., physician, certified nurse midwife, or nurse practitioner) or by the mother's documented and informed request. Prior to non-medically indicated supplementation, mothers will be informed of the risks of supplementing. The supplement should be fed to the baby by cup if possible and will be no more than 10$15 \mathrm{~mL}$ (per feeding) in a term baby (during the first 1-2 days of life). Alternative feeding methods such as syringe or spoon feeding may also be used; however, these methods have not been shown to be effective in preserving breastfeeding. Bottles will not be placed in or around the breastfeeding infant's bassinet. ${ }^{18-20}$

14. This institution does not give group instruction in the use of formula. Those parents who, after appropriate counseling, choose to formula feed their infants will be provided individual instruction.

15. Pacifiers will not be given to normal full-term breastfeeding infants. The pacifier guidelines at "name of institution" state that preterm infants in the Neonatal Intensive Care or Special Care Unit or infants with specific medical conditions (e.g., neonatal abstinence syndrome) may be given pacifiers for non-nutritive sucking. Newborns undergoing painful procedures (e.g., circumcision) may be given a pacifier as a method of pain management during the procedure. The infant will not return to the mother with the pacifier. "Name of institution" encourages "pain-free newborn care," which may include breastfeeding during the heel stick procedure for the newborn metabolic screening tests. ${ }^{21}$

16. Routine blood glucose monitoring of full-term healthy appropriate-for-gestational-age infants is not indicated. Assessment for clinical signs of hypoglycemia and dehydration will be ongoing. ${ }^{22}$

17. Antilactation drugs will not be given to any postpartum mother.

18. Routine use of nipple creams, ointments, or other topical preparations will be avoided unless such therapy has been indicated for a dermatologic problem. Mothers with sore nipples will be observed for latch-on techniques and will be instructed to apply expressed colostrum or breastmilk to the areola/nipple after each feeding.

19. Nipple shields or bottle nipples will not be routinely used to cover a mother's nipples, to treat latch-on problems, or to prevent or manage sore or cracked nipples or used when a mother has flat or inverted nipples. Nipple shields will be used only in conjunction 
with a lactation consultation and after other attempts to correct the difficulty have failed.

20. After 24 hours of life, if the infant has not latched-on or fed effectively, the mother will be instructed to begin to massage her breasts and hand express colostrum into the baby's mouth during feeding attempts. Skin-to-skin contact will be encouraged. Parents will be instructed to watch closely for feeding cues and whenever these are observed to awaken and feed the infant. If the baby continues to feed poorly, hand expression by the mother or a double set-up electric breast pump will be initiated and maintained approximately every 3 hours or a minimum of eight times per day. Any expressed colostrum or mother's milk will be fed to the baby by an alternative method. The mother will be reminded that she may not obtain much milk or even any milk the first few times she expresses her breasts. Until the mother's milk is available, a collaborative decision should be made among the mother, nurse, and healthcare professional (e.g., physician/nurse practitioner/certified nurse midwife) regarding the need to supplement the baby. Each day the responsible healthcare professional will be consulted regarding the volume and type of supplement. Pacifiers will be avoided. In cases of problem feeding, the lactation consultant or specialist will be consulted. ${ }^{14}$

21. If the baby is still not latching on well or feeding well when discharged to home, the feeding/expression/ supplementing plan will be reviewed in addition to routine breastfeeding instructions. A follow-up visit or contact will be scheduled within 24 hours. Depending on the clinical situation it may be appropriate to delay discharge of the couplet to provide further breastfeeding intervention, support, and education.

22. All babies should be seen for follow-up within the first few days postpartum. This visit should be with a physician (pediatrician or family physician) or other qualified healthcare practitioner for a formal evaluation of breastfeeding performance, a weight check, assessment of jaundice, and age-appropriate elimination: (a) For infants discharged at less than 2 days of age $(<48$ hours), follow-up at 2-4 days of age; and (b) for infants discharged between 48 and 72 hours, follow-up at 4-5 days of age. Infants discharged after 5-6 days may be seen 1 week later.

23. Mothers who are separated from their sick or premature infants will be:

a. Instructed on how to use skilled hand expression or the double set-up electric breast pump. Instructions will include expression at least eight times per day or approximately every 3 hours for 15 minutes (or until milk flow stops, whichever is greater) around the clock and the importance of not missing an expression session during the night.

b. Encouraged to breastfeed on demand as soon as the infant's condition permits

c. Taught proper storage and labeling of human milk and

d. Assisted in learning skilled hand expression or obtaining a double set-up electric breast pump prior to going home
24. Before leaving the hospital ${ }^{23}$ breastfeeding mothers should be able to

a. Position the baby correctly at the breast with no pain during the feeding

b. Latch the baby to breast properly

c. State when the baby is swallowing milk

d. State that the baby should be nursed a minimum of eight to 12 times a day until satiety, with some infants needing to be fed more frequently

e. State age-appropriate elimination patterns (at least six urinations per day and three to four stools per day by the fourth day of life)

f. List indications for calling a healthcare professional g. Manually express milk from their breasts

25. Prior to going home, mothers will be given the names and telephone numbers of community resources to contact for help with breastfeeding, including (the support group or resource recommended by "name of institution").

26. "Name of institution" does not accept free formula or free breastmilk substitutes. Nursery or Neonatal Intensive Care Unit discharge bags offered to all mothers will not contain infant formula, coupons for formula, logos of formula companies, or literature with formula company logos.

27. "Name of institution" health professionals will attend educational sessions on lactation management and breastfeeding promotion to ensure that correct, current, and consistent information is provided to all mothers wishing to breastfeed. ${ }^{24}$

\section{Application}

All breastfeeding patients.

\section{Exceptions}

Breastfeeding is contraindicated ${ }^{2,25}$ in the following situations:

- Mothers who are human immunodeficiency viruspositive in locations where artificial feeding is acceptable, feasible, affordable, sustainable, and safe ${ }^{26}$

- Mothers currently using illicit drugs (e.g., cocaine, heroin) unless specifically approved by the infant's healthcare provider on a case-by-case basis

- Mothers taking certain medications. Most prescribed and over-the-counter drugs are safe for the breastfeeding infant. Some medications may make it necessary to interrupt breastfeeding, such as radioactive isotopes, antimetabolites, cancer chemotherapy, some psychotropic medications, and a small number of other medications. The references used at "name of institution" are Medications and Mothers' Milk by T. Hale, ${ }^{27}$ the drugs and lactation database of the U.S. National Library of Medicine, TOXNET: Toxicology Data Network (LactMed), ${ }^{28}$ Breastfeeding: A Guide for the Medical Profession by R.A. Lawrence and R.M. Lawrence, ${ }^{29}$ Drugs in Pregnancy and Lactation by G.G. Briggs, R.K. Freeman, and S.J. Yaffe, ${ }^{30}$ and the American Academy of Pediatrics Statement on the Transfer of Drugs into Human Milk. ${ }^{31}$ (NB: Alternative local references and resources may be substituted if available.) 
- Mothers with active, untreated tuberculosis. A mother can express her milk until she is no longer contagious.

- Infants with galactosemia

- Mothers with active herpetic lesions on the breast(s). Breastfeeding can be recommended on the unaffected breast. (The Infectious Disease Service will be consulted for problematic infectious disease issues.)

- Mothers with onset of varicella within 5 days before or up to 48 hours after delivery, until she is no longer infectious

- Mothers with human T-cell lymphotropic virus type I or type II

\section{The Ten Steps to Successful Breastfeeding}

1. Have a written breastfeeding policy that is routinely communicated to all healthcare staff.

2. Train all health care staff in skills necessary to implement this policy.

3. Inform all pregnant women about the benefits and management of breastfeeding.

4. Help mothers initiate breastfeeding within 1 hour of birth.

5. Show mothers how to breastfeed and how to maintain lactation, even if they are separated from their infants.

6. Give newborn infants no food or drink other than breastmilk, unless medically indicated.

7. Practice rooming-in-allow mothers and infants to remain together-24 hours a day.

8. Encourage breastfeeding on demand.

9. Give no artificial teats or pacifiers to breastfeeding infants.

10. Foster the establishment of breastfeeding support groups and refer mothers to them, on discharge from the hospital or clinic.

\section{Other Related Policies}

- Policy \#

- Other references/resources ${ }^{32-35}$

\section{Initiated by}

List appropriate names, departments.

\section{Contributing Departments}

List all departments involved in developing policy.

\section{Research Needs}

Change in the hospital setting is hard. A comprehensive hospital breastfeeding policy that is clearly communicated to maternity staff may be a key step in the change process to support breastfeeding dyads. Rosenberg et al. ${ }^{36}$ reported that the presence of a written breastfeeding policy was independently associated with a statistically significant increase in the rate of breastfeeding.

Certain maternity care practices like The Ten Steps to Successful Breastfeeding (Table 2), the framework of the WHO-UNICEF Baby-Friendly Hospital Initiative, have been shown to influence breastfeeding outcomes. An analysis of the Infant Feeding Practices Study II (IFPS II) found that breastfeeding women who did not experience any of the Steps were 13 times more likely to stop breastfeeding early compared to those who experienced at least six Steps. In addition, the more steps practiced, the higher the duration and exclusivity of breastfeeding at 2 months. ${ }^{37}$ As only $8 \%$ of women surveyed in the IFPS II reported experiencing all six of the Baby-Friendly efforts measured, a great deal of work remains to be done.

Recommendations for further research include:

1. What are effective strategies to increase implementation of Baby-Friendly practices in the hospital setting?

2. How best to monitor staff adherence to a hospital's breastfeeding policy?

3. What are the effects of additional practices, not included in the original Ten Steps, on breastfeeding initiation and duration?

\section{Acknowledgments}

This work was supported in part by a grant from the Maternal and Child Health Bureau, U.S. Department of Health and Human Services.

\section{References}

1. U.S. Department of Health and Human Services. HHS Blueprint for Action on Breastfeeding. Office on Women's Health, U.S. Department of Health and Human Services, Washington, DC, 2000.

2. Gartner LM, Morton J, Lawrence RA, et al. Breastfeeding and the use of human milk. Pediatrics 2005;115:496-506.

3. Queenan JT, ed. ACOG Educational Bulletin Number 258. Breastfeeding: Maternal and Infant Aspects. Committees on Health Care for Underserved Women and Obstetric Practice, American College of Obstetricians and Gynecologists, Washington, DC, July 2000, pp. 1-16.

4. AAFP Breastfeeding Advisory Committee. Family Physicians Supporting Breastfeeding: Breastfeeding Position Paper 2008. www.aafp.org/online/en/home/policy/policies/ b/breastfeedingpositionpaper.html (accessed January 27, 2010).

5. World Health Organization, United Nations Children's Fund. Protecting, promoting and supporting breastfeeding: The special role of maternity services (a joint $\mathrm{WHO}$ / UNICEF statement). Int J Gynecol Obstet 1990; 31(Suppl 1): 171-183.

6. Academy of Breastfeeding Medicine Board of Directors. Position on breastfeeding. Breastfeed Med 2008;3:267-270.

7. WHO/UNICEF meeting on infant and young child feeding. J Nurse Midwifery 1980;25:31-38.

8. Innocenti Declaration on the Protection, Promotion and Support of Breastfeeding. UNICEF, New York, 1990.

9. World Health Organization, United Nations Children's Fund, Academy of Breastfeeding Medicine Board of Directors. Celebrating Innocenti 1990-2005: Achievements, Challenges and Future Imperatives. World Alliance for Breastfeeding Action. www.innocenti15.net/index.htm (accessed March 24, 2010).

10. United Nations Children's Fund, World Health Organization. Section 1. In: Baby Friendly Hospital Initiative: Revised, Updated and Expanded for Integrated Care. World Health Organization, UNICEF and Wellstart International, Geneva, 2009. 
11. Declercq E, Labbok MH, Sakala C, et al. Hospital practices and women's likelihood of fulfilling their intention to exclusively breastfeed. Am J Public Health 2009;99:929-935.

12. Merten S, Dratva J, Ackermann-Liebrich U. Do babyfriendly hospitals influence breastfeeding duration on a national level? Pediatrics 2005;116:e702-e708.

13. Academy of Breastfeeding Medicine Protocol Committee. Clinical protocol number \#19: Breastfeeding promotion in the prenatal setting. Breastfeed Med 2009;4:43-45.

14. Academy of Breastfeeding Medicine Protocol Committee. ABM clinical protocol \#3: Hospital guidelines for the use of supplementary feedings in the healthy term breastfed neonate, revised 2009. Breastfeed Med 2009;4:175-182.

15. Mikiel-Kostyra K, Mazur J, Boltruszko I. Effect of early skinto-skin contact after delivery on duration of breastfeeding: A prospective cohort study. Acta Paediatr 2002;91:1301-1306.

16. Righard L, Alade MO. Effect of delivery room routines on success of first breast-feed. Lancet 1990;336:1105-1107.

17. Eidelman AI, Hoffmann NW, Kaitz M. Cognitive deficits in women after childbirth. Obstet Gynecol 1993;81:764-767.

18. Howard CR, Howard FM, Lanphear B, et al. Randomized clinical trial of pacifier use and bottle-feeding or cupfeeding and their effect on breastfeeding. Pediatrics 2003;111:511-518.

19. Howard CR, de Blieck EA, ten Hoopen CB, et al. Physiologic stability of newborns during cup- and bottle-feeding. Pediatrics 1999;104:1204-1207.

20. Marinelli KA, Burke GS, Dodd VL. A comparison of the safety of cup feedings and bottle feedings in premature infants whose mothers intend to breastfeed. J Perinatol 2001; 21:350-355.

21. Gray L, Miller LW, Philipp BL, et al. Breastfeeding is analgesic in healthy newborns. Pediatrics 2002;109:590-593.

22. Wight N, Marinelli KA, Academy of Breastfeeding Medicine Protocol Committee. ABM clinical protocol \#1: Guidelines for glucose monitoring and treatment of hypoglycemia in breastfed neonates revision June, 2006. Breastfeed Med 2006;1:178-184

23. Academy of Breastfeeding Medicine Protocol Committee. ABM clinical protocol \#2 (2007 revision): Guidelines for hospital discharge of the breastfeeding term newborn and mother: "The going home protocol." Breastfeed Med 2007; 2:158-165.

24. American Academy of Pediatrics, American College of Obstetricians and Gynecologists. Breastfeeding Handbook for Physicians. American Academy of Pediatrics, Elk Grove Village, IL, 2006.

25. World Health Organization, UNICEF. Acceptable Medical Reasons for the Use of Breast-Milk Substitutes. World Health Organization, Geneva, 2009.

26. World Health Organization. HIV and Infant Feeding. 2007 http://www.who.int/hiv/pub/mtct/infant_feeding/en/ index.html (accessed June 8, 2010).

27. Hale TW. Medications and Mothers' Milk, $13^{\text {th }}$ ed. Hale Publishing, Amarillo, TX, 2008.
28. US National Library of Medicine. TOXNET: Toxicology Data Network. Drugs and Data Base (LactMed). toxnet.nlm.nih. gov/cgi-bin/sis/htmlgen?LACT (accessed January 27, 2010).

29. Lawrence RA, Lawrence RM. Breastfeeding: A Guide for the Medical Profession, $6^{\text {th }}$ ed. Mosby, Philadelphia, 2005.

30. Briggs GG, Freeman RK, Yaffe SJ. Drugs in Pregnancy and Lactation, $8^{\text {th }}$ ed. Williams and Wilkins, Baltimore, 2009.

31. Committee on Drugs, The American Academy of Pediatrics. The transfer of drugs and other chemicals into human milk. Pediatrics 2001;108:776-789.

32. American Academy of Pediatrics Section on Breastfeeding. Sample Hospital Breastfeeding Policy for Newborns, 2009. www.aap.org/bookstore (accessed January 27, 2010).

33. Academy of Breastfeeding Medicine Protocol Committee. ABM clinical protocol \#5: Peripartum breastfeeding management for the healthy mother and infant at term revision, June 2008. Breastfeed Med 2008;3:129-132.

34. Ip S, Chung M, Raman G, et al. Breastfeeding and Maternal and Infant Health Outcomes in Developed Countries. Evidence Report/Technology Assessment No. 153 (prepared by TuftsNew England Medical Center Evidence-Based Practice Center, under contract no. 290-02-0022). AHRQ Publication number 07-E007. Agency for Healthcare Research and Quality, Rockville, MD, 2007.

35. American Academy of Pediatrics. Redbook: 2009 Report of the Committee on Infectious Diseases, $28^{\text {th }}$ ed. American Academy of Pediatrics, Elk Grove, IL, 2009.

36. Rosenberg KD, Stull JD, Adler MR, et al. Impact of hospital policies on breastfeeding outcomes. Breastfeed Med 2008; 3:110-116.

37. DiGirolamo AM, Grummer-Strawn LM, Fein SB. Effect of maternity-care practices on breastfeeding. Pediatrics 2008; 122(Suppl 2):S43-S49.

$\mathrm{ABM}$ protocols expire 5 years from the date of publication. Evidence-based revisions are made within 5 years or sooner if there are significant changes in the evidence.

Contributor
*Barbara L. Philipp, M.D., FABM

Protocol Committee

Maya Bunik, M.D., MSPH, FABM

Caroline J. Chantry, M.D., FABM, Co-Chairperson

Cynthia R. Howard, M.D., MPH, FABM, Co-Chairperson

Ruth A. Lawrence, M.D., FABM

Kathleen A. Marinelli, M.D., FABM, Co-Chairperson

Larwrence Noble, M.D., FABM, Translations Chairperson

Nancy G. Powers, M.D., FABM

Julie Scott Taylor, M.D., M.Sc., FABM

*Lead author

For correspondence: abm@bfmed.org 
